\title{
On the $\psi_{2}$-behaviour of linear functionals on isotropic convex bodies
}

by

\author{
G. PAOURIS (Iraklion)
}

\begin{abstract}
The slicing problem can be reduced to the study of isotropic convex bodies $K$ with $\operatorname{diam}(K) \leq c \sqrt{n} L_{K}$, where $L_{K}$ is the isotropic constant. We study the $\psi_{2^{-}}$ behaviour of linear functionals on this class of bodies. It is proved that $\|\langle\cdot, \theta\rangle\|_{\psi_{2}} \leq C L_{K}$ for all $\theta$ in a subset $U$ of $S^{n-1}$ with measure $\sigma(U) \geq 1-\exp (-c \sqrt{n})$. However, there exist isotropic convex bodies $K$ with uniformly bounded geometric distance from the Euclidean ball, such that $\max _{\theta \in S^{n-1}}\|\langle\cdot, \theta\rangle\|_{\psi_{2}} \geq c \sqrt[4]{n} L_{K}$. In a different direction, we show that good average $\psi_{2}$-behaviour of linear functionals on an isotropic convex body implies very strong dimension-dependent concentration of volume inside a ball of radius $r \simeq \sqrt{n} L_{K}$.
\end{abstract}

1. Introduction. Let $K$ be a convex body in $\mathbb{R}^{n}$ with volume $|K|=1$ and centre of mass at the origin. Let $\alpha \in[1,2]$. For every bounded measurable function $f: K \rightarrow \mathbb{R}$, the $\alpha$-Orlicz norm of $f$ is defined by

$$
\|f\|_{\psi_{\alpha}}=\inf \left\{t>0: \int_{K} e^{|f / t|^{\alpha}} d x \leq 2\right\} .
$$

It is not hard to check that $\|f\|_{\psi_{\alpha}} \simeq \sup \left\{q^{-1 / \alpha}\|f\|_{q}: q \geq \alpha\right\}$. For every $x \in \mathbb{R}^{n}$ we consider the linear functional $f_{x}(y)=\langle y, x\rangle$. We say that $x$ defines a $\psi_{\alpha^{-}}$direction for $K$ with a constant $b>0$ if

$$
\left\|f_{x}\right\|_{\psi_{\alpha}} \leq b\left\|f_{x}\right\|_{\alpha}
$$

equivalently, if $\left\|f_{x}\right\|_{q} \leq c b q^{1 / \alpha}\left\|f_{x}\right\|_{\alpha}$ for every $q \geq \alpha$, where $c>0$ is an absolute constant. We say that $K$ is a $\psi_{\alpha}$-body with constant $b$ if (1.2) holds for every $x$.

It is clear that if $x$ defines a $\psi_{\alpha}$-direction for $K$ and if $T \in \mathrm{SL}(n)$, then $T^{*} x$ defines a $\psi_{\alpha}$-direction (with the same constant) for $T(K)$. It follows that $T(K)$ is a $\psi_{\alpha}$-body if $K$ is a $\psi_{\alpha}$-body. By Borel's lemma (see [14, Appendix III]), there exists an absolute constant $C>0$ with the following property: every convex body $K$ is a $\psi_{1}$-body with constant $C$.

2000 Mathematics Subject Classification: 52A20, 52A21, 52A40. 
The study of $\psi_{2}$-directions for linear functionals on convex bodies is motivated by the well known problem of the isotropic constant (see [13]). A convex body $K$ in $\mathbb{R}^{n}$ is said to be isotropic if it has volume $|K|=1$, centre of mass at the origin, and there exists a constant $L_{K}>0$ such that

$$
\int_{K}\langle y, \theta\rangle^{2} d y=L_{K}^{2}
$$

for every $\theta \in S^{n-1}$. Every convex body with centre of mass at the origin has a linear image which is isotropic. Moreover, this image is unique up to orthogonal transformations; this shows that the isotropic constant $L_{K}$ is well defined for the linear class of $K$.

The isotropic constant is closely related to the slicing problem which asks if there exists an absolute constant $c>0$ such that $\max _{\theta \in S^{n-1}}\left|K \cap \theta^{\perp}\right| \geq c$ for every convex body $K$ of volume 1 in $\mathbb{R}^{n}$ with centre of mass at the origin. Indeed, by Brunn's principle, for any convex body $K$ in $\mathbb{R}^{n}$ and any $\theta \in S^{n-1}$ the function $t \mapsto\left|K \cap\left(\theta^{\perp}+t \theta\right)\right|$ is log-concave on its support, and this implies that

$$
\int_{K}\langle y, \theta\rangle^{2} d y \simeq\left|K \cap \theta^{\perp}\right|^{-2} .
$$

Using this relation one can check (see [13]) that an affirmative answer to the slicing problem is equivalent to the following statement: There exists an absolute constant $C>0$ such that $L_{K} \leq C$ for every convex body $K$ of volume 1 with centre of mass at the origin.

One can easily see that $L_{K}=O(\sqrt{n})$ for every $K$. Uniform boundedness of $L_{K}$ is known for some classes of bodies: unit balls of spaces with 1-unconditional basis, zonoids and their polars, etc. The best known general upper estimate is due to Bourgain [7] (see [15] for the not necessarily symmetric case): $L_{K} \leq c \sqrt[4]{n} \log n$, where $c>0$ is an absolute constant. Bourgain's argument reduces the problem to the class of isotropic convex bodies $K$ with circumradius $R(K) \leq c \sqrt{n} L_{K}$, where $c>0$ is an absolute constant. The other main ingredient of the argument is the fact that every convex body is a $\psi_{1}$-body with a uniform constant. One can see from the proof that stronger information on the $\psi_{2}$-behaviour of linear functionals on $K$, combined with Talagrand's majorizing measure theorem and Pisier's $M M^{*}$-estimate, would result in an $O(\log n)$ estimate for $L_{K}$. In fact, Bourgain [8] has recently shown that the isotropic constant of a $\psi_{2}$-body with constant $b$ is bounded by $c b \log b$. In view of these observations, V. Milman asked the following question.

Question. Let $K$ be an isotropic convex body in $\mathbb{R}^{n}$. Is it true that most $\theta \in S^{n-1}$ define a $\psi_{2}$-direction for $K$ with a "good" constant (say, logarithmic in $n)$ ? 
In fact, it is not known if there exists an absolute constant $C>0$ such that every isotropic convex body has at least one $\psi_{2}$-direction with constant $C$. Bobkov and Nazarov (see [5] and [6]) have recently proved that if $K$ is an isotropic 1-unconditional convex body, then $\left\|f_{\theta}\right\|_{\psi_{2}} \leq c \sqrt{n}\|\theta\|_{\infty}$ for every $\theta \in S^{n-1}$. In particular, the diagonal direction is a $\psi_{2}$-direction. Zonoids form another class of convex bodies for which the existence of good $\psi_{2}$-directions can be established (see [16]). As for $\psi_{2}$-bodies, it is presently known that the unit balls of $\ell_{q}^{n}, 2 \leq q \leq \infty$, are $\psi_{2}$-bodies with a uniformly bounded constant $C$ (see [4] and [3]).

The aim of this paper is to study the $\psi_{2}$-behaviour of linear functionals on isotropic convex bodies $K$ with $R(K) \leq \gamma \sqrt{n} L_{K}$ in terms of the parameter $\gamma>0$. We call these "isotropic bodies with small diameter". Our general positive results are summarized in the following theorem.

Theorem A. Let $K$ be an isotropic convex body in $\mathbb{R}^{n}$. If $K$ is contained in $\left(\gamma \sqrt{n} L_{K}\right) B_{2}^{n}$ for some $\gamma>0$, then

$$
\sigma\left(\theta \in S^{n-1}:\left\|f_{\theta}\right\|_{\psi_{2}} \geq c_{1} \gamma t L_{K}\right) \leq \exp \left(-c_{2} \sqrt{n} t^{2} / \gamma\right)
$$

for every $t \geq 1$, where $c_{1}, c_{2}>0$ are absolute constants.

In other words, for a random direction $\theta \in S^{n-1}$, an isotropic convex body with small diameter satisfies

$$
\|\langle\cdot, \theta\rangle\|_{\psi_{2}} \leq C \gamma L_{K}
$$

However, the concentration estimate is rather weak (observe the $\sqrt{n}$ dependence in Theorem A) to be directly applicable to the slicing problem. It turns out that the positive result of Theorem A is optimal. One can construct an isotropic convex body $K$ with small diameter, for which $\max _{\theta}\left\|f_{\theta}\right\|_{\psi_{2}} \simeq$ $\sqrt[4]{n} L_{K}$. Surprisingly enough, the example may be given by a body whose geometric distance to the Euclidean ball is uniformly bounded.

THEOREM B. There exists an isotropic convex body of revolution $K$ in $\mathbb{R}^{n}$ with the following properties:

$$
c_{1} \sqrt{n} B_{2}^{n} \subseteq K \subseteq c_{2} \sqrt{n} B_{2}^{n}
$$

and

$$
\left\|\left\langle\cdot, e_{n}\right\rangle\right\|_{\psi_{2}} \geq c_{3} \sqrt[4]{n}
$$

where $c_{1}, c_{2}, c_{3}>0$ are absolute constants.

Theorems A and B are proved in Sections 2 and 3 respectively. In Section 4 we discuss the relation of the $\psi_{2}$-behaviour of linear functionals to a different question about the concentration of mass on isotropic convex bodies. Alesker [1] has proved that if $K$ is isotropic, then the Euclidean norm $f(y)=\|y\|_{2}$ satisfies the $\psi_{2}$-estimate $\|f\|_{\psi_{2}} \leq c\|f\|_{1} \leq c \sqrt{n} L_{K}$, where $c>0$ 
is an absolute constant. This leads to the tail estimate

$$
\operatorname{Prob}\left(\left\{y \in K:\|y\|_{2} \geq c_{1} t \sqrt{n} L_{K}\right\}\right) \leq 2 \exp \left(-t^{2}\right)
$$

for every $t>0$.

Bobkov and Nazarov [5] have obtained a striking dimension-dependent result in the 1-unconditional case: There exists an absolute constant $c>0$ such that if $K$ is an isotropic 1-unconditional convex body in $\mathbb{R}^{n}$, then

$$
\operatorname{Prob}\left(\left\{y \in K:\|y\|_{2} \geq c t \sqrt{n}\right\}\right) \leq \exp (-t \sqrt{n})
$$

for every $t \geq 1$. This estimate is stronger than (1.9) for all $t \geq 1$. A question which arises naturally and was actually stated in [5] is whether such an inequality may hold in full generality. This question is studied in [17] where equivalent conditions are provided. The results of [17] were recently used in [10] to establish a positive answer for a non-unconditional case: the unit balls of the Schatten trace classes $S_{p}^{n}, 1 \leq p \leq \infty$, of matrices on $\mathbb{R}^{n}$ or $\mathbb{C}^{n}$. Here we show that, in general, good average $\psi_{2}$-behaviour of linear functionals implies a very strong dimension-dependent concentration.

Theorem C. Let $K$ be an isotropic convex body in $\mathbb{R}^{n}$. Assume that

$$
\int_{S^{n-1}}\left\|f_{\theta}\right\|_{\psi_{2}} \sigma(d \theta) \leq \gamma L_{K}
$$

for some $\gamma>0$. Then

$$
\operatorname{Prob}\left(\left\{y \in K:\|y\|_{2} \geq c_{1} \gamma t \sqrt{n} L_{K}\right\}\right) \leq \exp \left(-c_{2} t \sqrt{n}\right)
$$

for every $t \geq 1$, where $c_{1}, c_{2}>0$ are absolute constants.

For the proof of Theorem $\mathrm{C}$ we study from an asymptotic point of view the family of $L_{q}$-centroid bodies of an isotropic convex body. In fact, this study was at the basis of our original proof of Theorem A. The possibility of giving the simpler proof in Section 2 was kindly pointed out to the author by G. Schechtman and B. Klartag. Nevertheless, the proof of Theorem C indicates that this line of thought has some interesting consequences.

Notation. We work in $\mathbb{R}^{n}$, which is equipped with a Euclidean structure $\langle\cdot, \cdot\rangle$. We denote by $\|\cdot\|_{2}$ the corresponding Euclidean norm, and write $B_{2}^{n}$ for the Euclidean unit ball and $S^{n-1}$ for the unit sphere. Volume is denoted by $|\cdot|$. We write $\sigma$ for the rotationally invariant probability measure on $S^{n-1}$ and $\omega_{n}$ for the volume of $B_{2}^{n}$.

A convex body is a compact convex subset $K$ of $\mathbb{R}^{n}$ with non-empty interior. We say that $K$ is symmetric (or centred) if $x \in K \Rightarrow-x \in K$. We say that $K$ has centre of mass at the origin if $\int_{K}\langle x, \theta\rangle d x=0$ for every $\theta \in S^{n-1}$. 
The circumradius of $K$ is the quantity $R(K)=\max \left\{\|x\|_{2}: x \in K\right\}$, and the polar body $K^{\circ}$ of $K$ is

$$
K^{\circ}:=\left\{y \in \mathbb{R}^{n}:\langle x, y\rangle \leq 1 \text { for all } x \in K\right\} .
$$

Let $V$ be a symmetric convex body in $\mathbb{R}^{n}$. The function $\|x\|_{V}=\inf \{\lambda>0$ : $x \in \lambda V\}$ is a norm on $\mathbb{R}^{n}$, and the space $\left(\mathbb{R}^{n},\|\cdot\|_{V}\right)$ will be denoted by $X_{V}$. The dual space of $X_{V}$ is $X_{V^{\circ}}$.

Whenever we write $a \simeq b$, we mean that there exist absolute constants $c_{1}, c_{2}>0$ such that $c_{1} a \leq b \leq c_{2} a$. The letters $c, c^{\prime}, C, c_{1}, c_{2}$ etc. denote absolute positive constants which may change from line to line. We refer to the books of Schneider [18] and Milman-Schechtman [14] for basic facts from the Brunn-Minkowski theory and the asymptotic theory of finite-dimensional normed spaces. A classical reference for isotropic convex bodies and the slicing problem is the paper [13] of Milman and Pajor.

Acknowledgements. The author is indebted to B. Klartag, R. Latała and G. Schechtman for suggestions and ideas which simplified and improved the original proofs of some results of this paper.

2. Isotropic convex bodies with small diameter. Let $K$ be an isotropic convex body in $\mathbb{R}^{n}$. In this section we assume $K \subseteq\left(\gamma \sqrt{n} L_{K}\right) B_{2}^{n}$ (one can easily check that $\gamma$ must exceed an absolute constant $c>0$ ). If $\gamma \simeq 1$ we say that $K$ is "an isotropic convex body with small diameter". Our aim is to show that a random $\theta \in S^{n-1}$ defines a $\psi_{2}$-direction with constant $C(\gamma)$. The precise statement is the following.

THEOREM 2.1. Let $K$ be an isotropic convex body in $\mathbb{R}^{n}$. Assume that for some $\gamma>0$ we have $K \subseteq\left(\gamma \sqrt{n} L_{K}\right) B_{2}^{n}$. Then

$$
\sigma\left(\theta \in S^{n-1}:\left\|f_{\theta}\right\|_{\psi_{2}} \geq c_{1} \gamma t L_{K}\right) \leq \exp \left(-c_{2} \sqrt{n} t^{2} / \gamma\right)
$$

for every $t \geq 1$, where $c_{1}, c_{2}>0$ are absolute constants.

The proof will be based on a well known consequence of the spherical isoperimetric inequality (see [14]): Let $n \geq 4$. If $F: S^{n-1} \rightarrow \mathbb{R}$ is a Lipschitz function with constant $b_{F}$, then

$$
\sigma\left(\theta \in S^{n-1}: F(\theta) \geq m_{F}+s\right) \leq \exp \left(-\frac{n s^{2}}{4 b_{F}^{2}}\right)
$$

for every $s>0$, where $m_{F}$ is the Lévy mean of $F$ (the unique $m>0$ for which $\sigma(\{F(\theta) \geq m\}) \geq 1 / 2$ and $\sigma(\{F(\theta) \leq m\}) \geq 1 / 2)$.

Consider the function $F(\theta)=\left\|f_{\theta}\right\|_{\psi_{2}}$ on $S^{n-1}$. In the next two claims we estimate the parameters $b_{F}$ and $m_{F}$.

Claim 2.2. We have $b_{F} \leq c_{3} \sqrt{\gamma} \sqrt[4]{n} L_{K}$, where $c_{3}>0$ is an absolute constant. 
Proof. Let $\theta \in S^{n-1}$. From the definition of $\|\cdot\|_{\psi_{\alpha}}$ we check that

$$
\left\|f_{\theta}\right\|_{\psi_{2}}^{2} \leq\left\|f_{\theta}\right\|_{\psi_{1}}\left\|f_{\theta}\right\|_{\infty}
$$

Since every $\theta \in S^{n-1}$ defines a $\psi_{1}$-direction with constant $C$ and $\left\|f_{\theta}\right\|_{\infty} \leq$ $R(K) \leq \gamma \sqrt{n} L_{K}$, we get

$$
\left\|f_{\theta}\right\|_{\psi_{2}}^{2} \leq\left(C L_{K}\right)\left(\gamma \sqrt{n} L_{K}\right)
$$

Then the claim follows from the triangle inequality for $\|\cdot\|_{\psi_{2}}$.

The next lemma will allow us to estimate $m_{F}$ (a similar argument appears in [16]).

LEMMA 2.3. Let $K$ be an isotropic convex body in $\mathbb{R}^{n}$ contained in $\left(\gamma \sqrt{n} L_{K}\right) B_{2}^{n}$. Then

$$
\int_{S^{n-1}} \int_{K} \exp \left(\frac{|\langle y, \theta\rangle|}{c_{4} \gamma L_{K}}\right)^{2} d y \sigma(d \theta) \leq 2,
$$

where $c_{4}>0$ is an absolute constant.

Proof. A direct computation shows that for every $p \geq 1$ and $y \in \mathbb{R}^{n}$,

$$
\left(\int_{S^{n-1}}|\langle y, \theta\rangle|^{p} \sigma(d \theta)\right)^{1 / p} \simeq\left(\frac{p}{p+n}\right)^{1 / 2}\|y\|_{2} .
$$

Let $c_{4}>0$ be a constant to be determined. We have

$$
\begin{aligned}
& \int_{S^{n-1}} \int_{K} \exp \left(\frac{|\langle y, \theta\rangle|}{c_{4} \gamma L_{K}}\right)^{2} d y \sigma(d \theta) \\
& =1+\sum_{k=1}^{\infty} \frac{1}{k ! c_{4}^{2 k} \gamma^{2 k} L_{K}^{2 k}} \int_{K} \int_{S^{n-1}}|\langle y, \theta\rangle|^{2 k} \sigma(d \theta) d y .
\end{aligned}
$$

Using (2.6) we see that the right hand side of (2.7) is bounded by

$$
1+\sum_{k=1}^{\infty} \frac{1}{k ! c_{4}^{2 k} \gamma^{2 k} L_{K}^{2 k}}\left(\frac{2 c_{5} k}{2 k+n}\right)^{k} \int_{K}\|y\|_{2}^{2 k} d y,
$$

where $c_{5}>0$ is an absolute constant. Since $\|y\|_{2} \leq \gamma \sqrt{n} L_{K}$ for every $y \in K$, we get

$$
\int_{S^{n-1}} \int_{K} \exp \left(\frac{|\langle y, \theta\rangle|}{c_{4} \gamma L_{K}}\right)^{2} d y \sigma(d \theta) \leq 1+\sum_{k=1}^{\infty}\left(\frac{2 e c_{5}^{2} n}{c_{4}^{2}(2 k+n)}\right)^{k} \leq 2
$$

if we choose $c_{4}=2 \sqrt{e} c_{5}$. 
An application of Markov's inequality shows that, with probability greater than or equal to $1 / 2$, a vector $\theta \in S^{n-1}$ satisfies

$$
\frac{1}{\left(c_{4} \gamma L_{K}\right)^{2 k}} \int_{K}\left|f_{\theta}(y)\right|^{2 k} d y \leq k ! \int_{K} \exp \left(\frac{\left|f_{\theta}(y)\right|}{c_{4} \gamma L_{K}}\right)^{2} d y \leq 4 k !
$$

Since $\left\|f_{\theta}\right\|_{\psi_{2}} \simeq \sup \left\{\left\|f_{\theta}\right\|_{2 k} / \sqrt{k}: k \in \mathbb{N}\right\}$, we have

$$
\sigma\left(\left\{\theta \in S^{n-1}:\left\|f_{\theta}\right\|_{\psi_{2}} \leq c_{6} \gamma L_{K}\right\}\right) \geq 1 / 2
$$

for some absolute constant $c_{6}>0$. This proves our next claim.

ClaIM 2.4. We have $m_{F} \leq c_{6} \gamma L_{K}$, with $c_{6}>0$ an absolute constant.

Proof of Theorem 2.1. We first observe that $\left\|f_{\theta}\right\|_{\psi_{2}} \geq c_{7}\left\|f_{\theta}\right\|_{2}=c_{7} L_{K}$ for every $\theta \in S^{n-1}$, and hence

$$
m_{F} \geq c_{7} L_{K}
$$

where $c_{7}>0$ is an absolute constant. Let $t \geq 1$. We apply (2.2) with $s=t m_{F}$, and using (2.12) and Claims 2.2 and 2.4 we get

$$
\begin{aligned}
\sigma\left(\theta \in S^{n-1}:\left\|f_{\theta}\right\|_{\psi_{2}} \geq c_{6}\right. & \left.\gamma(1+t) L_{K}\right) \\
& \leq \sigma\left(\theta \in S^{n-1}:\left\|f_{\theta}\right\|_{\psi_{2}} \geq(1+t) m_{F}\right) \\
& \leq \exp \left(-\frac{n t^{2} m_{F}^{2}}{4 b_{F}^{2}}\right) \leq \exp \left(-\frac{c_{7}^{2} n t^{2} L_{K}^{2}}{4 c_{3}^{2} \gamma \sqrt{n} L_{K}^{2}}\right) \\
& =\exp \left(-c_{2} \sqrt{n} t^{2} / \gamma\right),
\end{aligned}
$$

where $c_{2}=c_{7}^{2} /\left(4 c_{3}^{2}\right)$. Since $c_{6}(1+t) \leq 2 c_{6} t$, the result follows if we take $c_{1}=2 c_{6}$.

3. An example. In this section we exhibit an example of an isotropic convex body $K$ in $\mathbb{R}^{n}$ which has bounded geometric distance from the Euclidean unit ball but has the worst possible $\psi_{2}$-behaviour. One can build such an example inside the class of bodies of revolution.

LEMMA 3.1. There exist $a \simeq \sqrt{n}$ and $b \simeq 1 / \sqrt{n}$ such that the symmetric convex body

$$
C=\left\{y=(x, t):|t| \leq a,\|x\|_{2} \leq a-b|t|\right\}
$$

has volume 1 and satisfies

$$
c_{1} \leq \int_{C}\langle y, \theta\rangle^{2} d y \leq c_{2}
$$

for every $\theta \in S^{n-1}$, where $c_{1}, c_{2}>0$ are absolute constants. 
Proof. Let $r_{n-1}$ be the solution of the equation $\omega_{n-1} r^{n-1}=1$, and consider the body

$$
C_{1}=\left\{(x, t):|t| \leq r_{n-1},\|x\|_{2} \leq r_{n-1}-|t| / \sqrt{n}\right\} .
$$

Since $r_{n-1} \simeq \sqrt{n}$, we easily check that

$$
\begin{aligned}
\left|C_{1}\right| & =2 \omega_{n-1} \int_{0}^{r_{n-1}}\left(r_{n-1}-\frac{t}{\sqrt{n}}\right)^{n-1} d t \\
& =2 \omega_{n-1} r_{n-1}^{n} \sqrt{n} \frac{1-(1-1 / \sqrt{n})^{n}}{n} \simeq 1 .
\end{aligned}
$$

Therefore, we may find $s>0$ with $s^{n} \simeq 1$ such that $C:=s C_{1}$ has volume 1 . It is clear that $C$ can be written in the form (3.1), with $a=s r_{n-1} \simeq \sqrt{n}$ and $b=1 / \sqrt{n}$.

Also, $C$ is symmetric with respect to the coordinate subspaces, and hence (3.2) will hold for every $\theta \in S^{n-1}$ provided that

$$
c_{1} \leq \int_{C}\left\langle y, e_{j}\right\rangle^{2} d y \leq c_{2} .
$$

From (1.4) it is enough to check that

$$
c_{3} \leq\left|C \cap e_{j}^{\perp}\right| \leq c_{4}
$$

for every $j=1, \ldots, n$. Let $j \leq n-1$. Then

$$
\begin{aligned}
\left|C_{1} \cap e_{j}^{\perp}\right| & =2 \omega_{n-2} \int_{0}^{r_{n-1}}\left(r_{n-1}-\frac{t}{\sqrt{n}}\right)^{n-2} d t \\
& \leq 2 \omega_{n-2}\left(\int_{0}^{r_{n-1}}\left(r_{n-1}-\frac{t}{\sqrt{n}}\right)^{n-1} d t\right)^{(n-2) /(n-1)} \sqrt[n-1]{r_{n-1}} \\
& =2 \omega_{n-2}\left(\frac{\left|C_{1}\right|}{2 \omega_{n-1}}\right)^{(n-2) /(n-1)} \sqrt[n-1]{r_{n-1}} \leq c_{5} \sqrt[n-1]{r_{n-1}} \simeq 1 .
\end{aligned}
$$

For the lower bound we observe that

$$
\begin{aligned}
\left|C_{1} \cap e_{j}^{\perp}\right| & =2 \omega_{n-2} \int_{0}^{r_{n-1}}\left(r_{n-1}-\frac{t}{\sqrt{n}}\right)^{n-2} d t \\
& \geq \frac{\omega_{n-2}}{r_{n-1} \omega_{n-1}} 2 \omega_{n-1} \int_{0}^{r_{n-1}}\left(r_{n-1}-\frac{t}{\sqrt{n}}\right)^{n-1} d t=\frac{\omega_{n-2}}{r_{n-1} \omega_{n-1}} \simeq 1 .
\end{aligned}
$$

Finally,

$$
\left|C_{1} \cap e_{n}^{\perp}\right|=\omega_{n-1} r_{n-1}^{n-1}=1 .
$$

Since $\left|C \cap e_{j}^{\perp}\right|=s^{n-1}\left|C_{1} \cap e_{j}^{\perp}\right| \simeq\left|C_{1} \cap e_{j}^{\perp}\right|$ for all $j \leq n$, the lemma is proved. 
Starting with $C$, we easily pass to a "similar" isotropic body.

Lemma 3.2. There exist $a_{1}, R_{1} \simeq \sqrt{n}$ and $b_{1} \simeq 1 / \sqrt{n}$ such that the symmetric convex body

$$
K=\left\{y=(x, t):|t| \leq R_{1},\|x\|_{2} \leq a_{1}-b_{1}|t|\right\}
$$

is isotropic.

Proof. Consider the body $C$ of the previous lemma. We first observe (see [13, equation (1.9) on p. 71]) that

$$
L_{C}^{2 n}=\prod_{j=1}^{n} \int_{C}\left\langle y, e_{j}\right\rangle^{2} d y .
$$

Because of (3.2), this implies that $L_{C} \simeq 1$. By the symmetries of $C$ there exists a diagonal operator $T=\operatorname{diag}(u, \ldots, u, v)$ such that $K=T(C)$ is isotropic. For every $\theta \in S^{n-1}$ we have

$$
1 \simeq L_{C}^{2}=L_{K}^{2}=\int_{K}\langle x, \theta\rangle^{2} d x=\int_{C}\langle x, T \theta\rangle^{2} d x \simeq\|T \theta\|_{2}^{2} .
$$

This shows that $u \simeq 1$ and $v \simeq 1$. Finally, $K$ can be written in the form (3.8) with $R_{1}=a v, a_{1}=a u$ and $b_{1}=b u / v$. This completes the proof.

LEMma 3.3. Let $K$ be the body in Lemma 3.2. If $0<s \leq c_{1} \sqrt{n}$, then

$$
\operatorname{Prob}(\{(x, t):|t| \geq s\}) \geq c_{2} \exp \left(-c_{3} s\right),
$$

where $c_{1}, c_{2}, c_{3}>0$ are absolute constants.

Proof. Direct computation shows that

$$
\begin{aligned}
\operatorname{Prob}(\{(x, t):|t| \geq s\}) & =2 \omega_{n-1} \int_{s}^{R_{1}}\left(a_{1}-b_{1} t\right)^{n-1} d t \\
& =2 \omega_{n-1} \frac{a_{1}^{n}}{n b_{1}}\left(\left(1-\frac{b_{1} s}{a_{1}}\right)^{n}-\left(1-\frac{b_{1} R_{1}}{a_{1}}\right)^{n}\right) .
\end{aligned}
$$

Since $\omega_{n-1} a_{1}^{n-1}=\left|K \cap e_{n}^{\perp}\right| \simeq 1$ and $a_{1} /\left(n b_{1}\right) \simeq 1$, we see that

$$
2 \omega_{n-1} \frac{a_{1}^{n}}{n b_{1}} \geq c_{4}
$$

for some absolute constant $c_{4}>0$. If $s \leq a_{1} /\left(2 b_{1}\right) \simeq n$, using the numerical inequality $1-x \geq e^{-2 x}$ for $x \in[0,1 / 2]$, we obtain

$$
\left(1-\frac{b_{1} s}{a_{1}}\right)^{n} \geq \exp \left(-2 b_{1} n s / a_{1}\right) \geq \exp \left(-c_{3} s\right) .
$$

On the other hand,

$$
\left(1-\frac{b_{1} R_{1}}{a_{1}}\right)^{n} \leq \exp \left(-b_{1} R_{1} n / a_{1}\right) \leq \exp \left(-c_{5} \sqrt{n}\right) .
$$


If $s \leq c_{1} \sqrt{n}$ for a suitable absolute constant $c_{1}>0$, then

$$
\exp \left(-c_{5} \sqrt{n}\right) \leq \frac{1}{2} \exp \left(-c_{3} s\right)
$$

It follows that if $s \leq c_{1} \sqrt{n}$, then

$$
\operatorname{Prob}(\{(x, t):|t| \geq s\}) \geq\left(c_{4} / 2\right) \exp \left(-c_{3} s\right) .
$$

This gives the result, with $c_{2}=c_{4} / 2$.

Lemma 3.4. Let $K$ be a convex body of volume 1 in $\mathbb{R}^{n}$. If $q \geq 1$, then

$$
\operatorname{Prob}\left(y \in K:|\langle y, \theta\rangle| \geq \alpha\left\|f_{\theta}\right\|_{q} s\right) \leq e^{-q s}
$$

for all $\theta \in S^{n-1}$ and $s \geq 1$, where $\alpha>0$ is an absolute constant.

Proof. Let $q \geq 1$ and $\theta \in S^{n-1}$. Markov's inequality shows that

$$
\operatorname{Prob}\left(y \in K:|\langle y, \theta\rangle| \geq e^{3}\left\|f_{\theta}\right\|_{q}\right) \leq e^{-3 q} \text {. }
$$

Since $y \mapsto|\langle y, \theta\rangle|$ is a seminorm, from Borel's lemma (see [14, Appendix III]) we get

$$
\begin{aligned}
& \operatorname{Prob}\left(y \in K:|\langle y, \theta\rangle| \geq e^{3}\left\|f_{\theta}\right\|_{q} s\right) \\
& \quad \leq\left(1-e^{-3 q}\right)\left(\frac{e^{-3 q}}{1-e^{-3 q}}\right)^{(s+1) / 2} \leq e^{-q s}
\end{aligned}
$$

for every $s \geq 1$. This proves the lemma with $\alpha=e^{3}$.

REMARK 3.5. The same lemma is proved in [17] as a consequence of a much more general optimal $L^{q}$-norm inequality of Carbery and Wright (see [9]) for polynomials over convex bodies. The simple argument which is presented here was kindly communicated to the author by R. Latala.

Let $K$ be the body from Lemma 3.2. The next two claims describe two "contradictory" properties of $K$.

Claim 3.6. We have $c_{1} \sqrt{n} B_{2}^{n} \subseteq K \subseteq c_{2} \sqrt{n} B_{2}^{n}$, where $c_{1}, c_{2}>0$ are absolute constants.

Proof. The problem is two-dimensional. For every $y=(x, t) \in K$ we have

$$
\|y\|_{2}^{2}=\|x\|_{2}^{2}+t^{2} \leq a_{1}^{2}+R_{1}^{2} \leq c_{2}^{2} n
$$

where $c_{2}>0$ is an absolute constant, because $a_{1}, R_{1} \simeq \sqrt{n}$. This shows that $K \subseteq c_{2} \sqrt{n} B_{2}^{n}$. For the other inclusion, we observe that the inradius of $K$ is equal to $\min \left\{R_{1}, d\right\}$, where $d$ is the distance from $(0,0)$ to the line $y=a_{1}-b_{1} t$ in $\mathbb{R}^{2}$. We have

$$
d=\frac{a_{1}}{\sqrt{1+b_{1}^{2}}} \simeq \sqrt{n},
$$

and hence $K \supseteq c_{1} \sqrt{n} B_{2}^{n}$ for some absolute constant $c_{1}>0$. 
Claim 3.7. We have $\left\|\left\langle\cdot, e_{n}\right\rangle\right\|_{L^{\psi_{2}(K)}} \geq c \sqrt[4]{n}$, where $c>0$ is an absolute constant.

Proof. Let $q \geq 1$. If $\alpha\left\|\left\langle\cdot, e_{n}\right\rangle\right\|_{q} \leq c_{1} \sqrt{n}$, Lemmas 3.3 and 3.4 show that

$$
\begin{aligned}
c_{2} \exp \left(-c_{3} \alpha\left\|\left\langle\cdot, e_{n}\right\rangle\right\|_{q}\right) \\
\quad \leq \operatorname{Prob}\left(\left\{y \in K:\left|\left\langle y, e_{n}\right\rangle\right| \geq \alpha\left\|\left\langle\cdot, e_{n}\right\rangle\right\|_{q}\right\}\right) \leq e^{-q} .
\end{aligned}
$$

For these values of $q$ it follows that

$$
c_{3} \alpha\left\|\left\langle\cdot, e_{n}\right\rangle\right\|_{q} \geq q+\log c_{2} .
$$

Since $e_{n}$ defines a $\psi_{1}$-direction for $K$, we have

$$
\left\|\left\langle\cdot, e_{n}\right\rangle\right\|_{q} \leq c_{4} q\left\|\left\langle\cdot, e_{n}\right\rangle\right\|_{1} \leq c_{5} q .
$$

Therefore, we can find $q_{*} \simeq \sqrt{n}$ such that $\alpha\left\|\left\langle\cdot, e_{n}\right\rangle\right\|_{q_{*}} \leq c_{1} \sqrt{n}$. Going back to (3.23) we see that

$$
\left\|\left\langle\cdot, e_{n}\right\rangle\right\|_{\psi_{2}} \geq c_{6} \frac{\left\|\left\langle\cdot, e_{n}\right\rangle\right\|_{q_{*}}}{\sqrt{q_{*}}} \geq c \sqrt[4]{n}
$$

for some absolute constant $c>0$.

Lemma 3.2 and Claims 3.6 and 3.7 are summarized in the next theorem.

THEOREM 3.8. There exists an isotropic convex body of revolution $K$ in $\mathbb{R}^{n}$ with the following properties:

$$
c_{1} \sqrt{n} B_{2}^{n} \subseteq K \subseteq c_{2} \sqrt{n} B_{2}^{n}
$$

and

$$
\left\|\left\langle\cdot, e_{n}\right\rangle\right\|_{\psi_{2}} \geq c_{3} \sqrt[4]{n}
$$

where $c_{1}, c_{2}, c_{3}>0$ are absolute constants.

4. $L_{q}$-centroid bodies. Let $K$ be a convex body of volume 1 in $\mathbb{R}^{n}$ and let $q \geq 1$. The $L_{q}$-centroid body $Z_{q}(K)$ of $K$ is the symmetric convex body whose support function is given by

$$
N_{q}(K, x):=\left\|f_{x}\right\|_{q}=\left(\int_{K}|\langle y, x\rangle|^{q} d y\right)^{1 / q} .
$$

It is easy to check that $Z_{p}(K) \subseteq Z_{q}(K) \subseteq Z_{\infty}(K)$ for every $1 \leq p \leq q \leq \infty$, where $Z_{\infty}(K)=\widehat{K}:=\operatorname{co}\{K,-K\}$. If $K$ has its centre of mass at the origin, then $Z_{q}(K) \simeq \widehat{K}$ for all $q \geq n$. This is a consequence of the Brunn-Minkowski inequality (for a proof, see [16]). $L_{q}$-centroid bodies were introduced in [12] under a different normalization. 
For every $p, q \geq 1$ we define

$$
\begin{aligned}
A_{q}(K) & =\int_{S^{n-1}} N_{q}(K, \theta) \sigma(d \theta), \\
A_{q, p}(K) & =\left(\int_{S^{n-1}} N_{q}^{p}(K, \theta) \sigma(d \theta)\right)^{1 / p} .
\end{aligned}
$$

The next proposition describes the behaviour of $p \mapsto A_{q, p}(K)$ for a fixed value of $q$.

Proposition 4.1. Let $K$ be a convex body of volume 1 in $\mathbb{R}^{n}$ with centre of mass at the origin. For every $p, q \in[1, n]$ we have

$$
A_{q, p}(K) \simeq \max \left\{A_{q}(K), \frac{R\left(Z_{q}(K)\right) \sqrt{p}}{\sqrt{n}}\right\} .
$$

If $p \geq n$, then $A_{q, p}(K) \simeq R\left(Z_{q}(K)\right)$.

Proof. Let $V$ be a symmetric convex body in $\mathbb{R}^{n}$, and let $\|\cdot\|$ be the norm on $\mathbb{R}^{n}$ induced by $V$. For every $p \geq 1$, let

$$
M_{p}:=M_{p}(V)=\left(\int_{S^{n-1}}\|\theta\|^{p} \sigma(d \theta)\right)^{1 / p} .
$$

The behaviour of $p \mapsto M_{p}$ was clarified by Litvak, Milman and Schechtman in [11]: If $R=\max \left\{\|x\|_{2}: x \in V^{\circ}\right\}$ then

$$
M_{p} \simeq \max \left\{M_{1}, \frac{R \sqrt{p}}{\sqrt{n}}\right\}
$$

for all $p \in[1, n]$, and $M_{p} \simeq R$ if $p \geq n$. A direct application to the polar body of $Z_{q}(K)$ gives the result.

Proposition 4.2. There exist $c_{1}, c_{2}>0$ and $n_{0} \in \mathbb{N}$ with the following property: if $n \geq n_{0}$ and $K$ is an isotropic convex body in $\mathbb{R}^{n}$ then, for every $2 \leq q \leq c_{1} \sqrt{n}$,

$$
I_{q}(K):=\left(\int_{K}\|y\|_{2}^{q} d y\right)^{1 / q} \leq c_{2} \sqrt{n} \frac{A_{q}(K)}{\sqrt{q}} .
$$

For the proof we will need two simple observations.

Claim 4.3. Let $K$ be an isotropic convex body in $\mathbb{R}^{n}$. For every $2 \leq q \leq n$ we have

$$
R\left(Z_{q}(K)\right) \leq c_{3} q L_{K}
$$

and

$$
A_{q, q}(K) \simeq \sqrt{q / n} I_{q}(K) \geq c_{4} \sqrt{q} L_{K},
$$

where $c_{3}, c_{4}>0$ are absolute constants. 
Proof. The first assertion follows from the fact that $K$ is a $\psi_{1}$-body. For the second we use (2.6) and integration over $K$. The last inequality is a direct consequence of Hölder's inequality: we have $I_{q}(K) \geq I_{2}(K)=\sqrt{n} L_{K}$.

Proof of Proposition 4.2. Observe that $Z_{2}(K)=L_{K} B_{2}^{n}$, which implies $R\left(Z_{2}(K)\right)=A_{2}(K)=L_{K}$. Also, when $q \rightarrow \infty$, both $R\left(Z_{q}(K)\right)$ and $A_{q}(K)$ tend to $R(K)$. So, if we define

$$
N(K):=\left\{q \geq 2: R\left(Z_{q}(K)\right) \sqrt{q}<\sqrt{n} A_{q}(K)\right\},
$$

we immediately see that $2 \in N(K)$ and $N(K)$ is bounded.

We set $q_{*}:=\sup \{q \geq 2:[2, q] \subseteq N(K)\}$. Then $q_{*}$ is well defined and the continuity of $R\left(Z_{q}(K)\right)$ and $A_{q}(K)$ with respect to $q$ shows that

$$
R\left(Z_{q_{*}}(K)\right) \sqrt{q_{*}}=\sqrt{n} A_{q_{*}}(K) .
$$

From Proposition 4.1, this last equation shows that

$$
A_{q_{*}, q_{*}}(K) \simeq A_{q_{*}}(K) .
$$

Going back to (4.11) and using Claim 4.3 we get

$$
q_{*} \geq c_{1} \sqrt{n} \text {. }
$$

Finally, since $\left[2, c_{1} \sqrt{n}\right] \subseteq(K)$, for every $2 \leq q \leq c_{1} \sqrt{n}$ we have $A_{q, q}(K) \simeq$ $A_{q}(K)$ and this implies that

$$
I_{q}(K) \simeq \sqrt{n / q} A_{q, q}(K) \leq c_{2} \sqrt{n} \frac{A_{q}(K)}{\sqrt{q}}
$$

for some absolute constant $c_{2}>0$.

We can now give a proof of Theorem $\mathrm{C}$ based on the following criterion from [17].

Lemma 4.4. Let $\delta \geq 1$ and let $1 \ll \phi(n) \ll n$ be a positive constant. For every isotropic convex body $K$ in $\mathbb{R}^{n}$, the following statements are equivalent:

(a) For every $t \geq 1$,

$$
\operatorname{Prob}\left(\left\{y \in K:\|y\|_{2} \geq \delta t \sqrt{n} L_{K}\right\}\right) \leq \exp (-\phi(n) t) .
$$

(b) For every $2 \leq q \leq c \phi(n)$,

$$
I_{q}(K)=\left(\int_{K}\|y\|_{2}^{q} d y\right)^{1 / q} \leq c(\delta) \sqrt{n} L_{K},
$$

where $c(\delta) \simeq \delta$.

Proof of Theorem $C$. We assume that $K$ is an isotropic convex body in $\mathbb{R}^{n}$ which satisfies

$$
\int_{S^{n-1}}\left\|f_{\theta}\right\|_{\psi_{2}} \sigma(d \theta) \leq \gamma L_{K}
$$


for some $\gamma>0$. Since $\left\|f_{\theta}\right\|_{q} \leq c_{3} \sqrt{q}\left\|f_{\theta}\right\|_{\psi_{2}}$ for every $q \geq 2$, we get

$$
A_{q}(K)=\int_{S^{n-1}}\left\|f_{\theta}\right\|_{q} \sigma(d \theta) \leq c_{4} \gamma \sqrt{q} L_{K}
$$

for every $q \geq 2$, where $c_{4}>0$ is an absolute constant. Now, Proposition 4.2 shows that

$$
I_{q}(K) \leq c_{5} \gamma \sqrt{n} L_{K}
$$

for every $2 \leq q \leq c_{6} \sqrt{n}$, where $c_{5}, c_{6}>0$ are absolute constants. Then, we can apply Proposition 4.4 with $\delta \simeq \gamma$ and $\phi(n) \simeq \sqrt{n}$ to get

$$
\operatorname{Prob}\left(\left\{y \in K:\|y\|_{2} \geq c_{1} \gamma t \sqrt{n} L_{K}\right\}\right) \leq \exp \left(-c_{2} t \sqrt{n}\right)
$$

for every $t \geq 1$, where $c_{1}, c_{2}>0$ are absolute constants.

\section{References}

[1] S. Alesker, $\psi_{2}$-estimate for the Euclidean norm on a convex body in isotropic position, in: Geometric Aspects of Functional Analysis, J. Lindenstrauss and V. D. Milman (eds.), Oper. Theory Adv. Appl. 77, Birkhäuser, Basel, 1995, 1-4.

[2] M. Anttila, K. Ball and I. Perissinaki, The central limit problem for convex bodies, Trans. Amer. Math. Soc. 355 (2003), 4723-4735; erratum, ibid. 365 (2004), 2137.

[3] F. Barthe, O. Guédon, S. Mendelson and A. Naor, A probabilistic approach to the geometry of the $\ell_{p}^{n}$-ball, Ann. Probab., to appear.

[4] F. Barthe and A. Koldobsky, Extremal slabs in the cube and the Laplace transform, Adv. Math. 174 (2003), 89-114.

[5] S. G. Bobkov and F. L. Nazarov, On convex bodies and log-concave probability measures with unconditional basis, in: Geometric Aspects of Functional Analysis, V. D. Milman and G. Schechtman (eds.), Lecture Notes in Math. 1807, Springer, Berlin, 2003, 53-69.

[6] S. G. Bobkov and F. L. Nazarov, Large deviations of typical linear functionals on a convex body with unconditional basis, in: Stochastic Inequalities and Applications, Progr. Probab. 56, Birkhäuser, Basel, 2003, 3-13.

[7] J. Bourgain, On the distribution of polynomials on high dimensional convex sets, in: Geometric Aspects of Functional Analysis, J. Lindenstrauss and V. D. Milman (eds.), Lecture Notes in Math. 1469, Springer, Berlin, 1991, 127-137.

[8] -, On the isotropy-constant problem for "PSI-2"-bodies, in: Geometric Aspects of Functional Analysis, V. D. Milman and G. Schechtman (eds.), Lecture Notes in Math. 1807, Springer, Berlin, 2003, 114-121.

[9] A. Carbery and J. Wright, Distributional and $L^{q}$-norm inequalities for polynomials over convex bodies in $\mathbb{R}^{n}$, Math. Res. Lett. 8 (2001), 233-248.

[10] O. Guédon and G. Paouris, Concentration of mass on the Schatten classes, preprint.

[11] A. E. Litvak, V. D. Milman and G. Schechtman, Averages of norms and quasinorms, Math. Ann. 312 (1998), 95-124.

[12] E. Lutwak and G. Zhang, Blaschke-Santaló inequalities, J. Differential Geom. 47 (1997), 1-16.

[13] V. D. Milman and A. Pajor, Isotropic position and inertia ellipsoids and zonoids of the unit ball of a normed n-dimensional space, in: Geometric Aspects of Functional 
Analysis, J. Lindenstrauss and V. D. Milman (eds.), Lecture Notes in Math. 1376, Springer, Berlin, 1989, 64-104.

[14] V. D. Milman and G. Schechtman, Asymptotic Theory of Finite Dimensional Normed Spaces, Lecture Notes in Math. 1200, Springer, Berlin, 1986.

[15] G. Paouris, On the isotropic constant of non-symmetric convex bodies, in: Geometric Aspects of Functional Analysis, V. D. Milman and G. Schechtman (eds.), Lecture Notes in Math. 1745, Springer, Berlin, 2000, 239-243.

[16] -, $\Psi_{2}$-estimates for linear functionals on zonoids, in: Geometric Aspects of Functional Analysis, V. D. Milman and G. Schechtman (eds.), Lecture Notes in Math. 1807, Springer, Berlin, 2003, 211-222.

[17] - Concentration of mass and central limit properties of isotropic convex bodies, Proc. Amer. Math. Soc. 133 (2005), 565-575.

[18] R. Schneider, Convex Bodies: The Brunn-Minkowski Theory, Encyclopedia Math. Appl. 44, Cambridge Univ. Press, Cambridge, 1993.

Department of Mathematics

University of Crete

Iraklion 714-09, Crete, Greece

E-mail: paouris@math.uoc.gr 